\title{
Resistência a antimicrobianos em Staphylococcus aureus isolados de mastite em bovinos leiteiros de Minas Gerais, Brasil
}

\author{
Antimicrobial resistance in Staphylococcus aureus isolated \\ from mastitis in dairy herds from the state of Minas Gerais, Brazil \\ Geraldo Márcio da Costa * ${ }^{1 *}$ Rodrigo Alves Barros', Dircéia Aparecida da Costa Custódio', \\ Ulisses de Pádua Pereira', Demétrio Junqueira Figueiredo', Nivaldo da Silva²
}

| | | | | | | | | | | | | | | | | | | | | | | | | | | | | | | | | | | | | | | | | | | | | | | | | | | | | | | | | | | | | | | | | | | | | | | | | | | | | | | | | | | | | | | | | | | | | | | | | | | | | | | | | | | | | | | | | | | | | | | | | | | | | | | | | | | | | | | | | | | | | | | | | | | | | | | | | | | | | | | | | | | | | | | | | | | | | | | | | | | | | | | | | | | | | | | | | | | | | | | | | | | | | | | | | |

RESUMO: Staphylococcus aureus (S. aureus) destaca-se como um dos agentes etiológicos mais frequentes da mastite bovina, que causa os maiores prejuízos econômicos à pecuária leiteira brasileira. Visando avaliar o perfil de sensibilidade deste agente aos antimicrobianos, 352 isolados provenientes de 35 rebanhos leiteiros localizados em Minas Gerais foram submetidos a testes de antibiograma, utilizando-se a técnica de difusão em disco. Nitrofurantoína, bem como as associaçôes de neomicina, bacitracina e tetraciclina (NBT) e penicilina, nafcilina e dihidroestreptomicina (PND), apresentaram 100\% de eficiência in vitro. Foram verificados baixos índices de resistência no grupo das cefalosporinas, com valores de 0, 0,28 e 0,40\% para cefquimona, cefalotina e ceftiofur, respectivamente. Entre os aminoglicosídeos, observou-se 1,69\% de resistência para gentamicina e 3,35\% para a neomicina. $\mathrm{O}$ maior índice de resistência foi observado para polimixina B (82\%), seguido pelos betalactâmicos, ampicilina e penicilina, com índices de resistência de 80,92 e 80,45\%, respectivamente. Níveis intermediários de resistência foram observados para tetraciclina, lincomicina, cefoperazona e sulfazotrim. Entre os isolados testados, $65(18,15 \%)$, oriundos de 24 dentre os 35 rebanhos estudados, apresentaram multirresistência (índice MAR $\geq 0,2$ ). Os resultados apontaram grande variação nos perfis de resistência aos antimicrobianos, assim como a ocorrência de múltipla resistência entre algumas cepas estudadas, salientando a necessidade de testes de antibiograma para a escolha dos antimicrobianos mais adequados para o tratamento ou prevenção de mastite causada por $S$. aureus.

PALAVRAS-CHAVE: Staphylococci; antibióticos; suscetibilidade; tratamento.

\begin{abstract}
Staphylococcus aureus ( $S$. aureus) stands out as one of the most common etiological agents of bovine mastitis, currently causing major economic losses to dairy farming. In order to evaluate the sensitivity of this micro-organism to antimicrobials, 352 isolates from 35 dairy herds located in the state of Minas Gerais were submitted to antibiogram tests, using the disc diffusion technique. Nitrofurantoin and the associations of neomycin, bacitracin and tetracycline (NBT), as well as penicillin, nafcillin and dihydrostreptomycin (PND), showed $100 \%$ efficiency in vitro. There were low resistance rates in the group of cephalosporins, with values of $0,0.28$ and $0.40 \%$ for cefquimona, cephalothin, and ceftiofur, respectively. Among the aminoglycosides, there was $1.69 \%$ resistance to gentamicin and $3.35 \%$ for neomycin. The highest resistance was observed for polymyxin B (82\%), followed by betalactams, ampicillin and penicillin, with resistance rates of 80.92 and $80.45 \%$, respectively. Moderate levels of resistance were observed for tetracycline, lincomycin, cefoperazone and sulfazotrim. Among the tested isolates, 65 (18.15\%), coming from 24 of the 35 herds studied, showed multidrug resistance (MAR index $\geq 0.2$ ). The results pointed out to high variation in antimicrobial resistance profiles and the occurrence of multidrug resistance among some studied strains, highlighting the importance of antibiogram tests for the choice of an appropriate drug to be used for the treatment or prevention of mastitis caused by $S$. aureus.
\end{abstract}

KEYWORDS: Staphylococci; antibiotics; susceptibility; treatment. 


\section{INTRODUÇÃO}

A mastite bovina é uma afecçáo de cunho multifatorial que tem nas inter-relaçôes entre o hospedeiro, o ambiente e os agentes infecciosos os fatores determinantes para sua ocorrência (LE BLANK et al., 2006). Apesar de todos os avanços logrados nas últimas décadas em termos de prevençáo e controle, esta enfermidade permanece como a doença infecciosa mais prevalente e economicamente relevante entre bovinos leiteiros em todos os continentes (Aarestrup; Jensen, 1997; Bradley, 2002; Vintov et al., 2003).

O impacto decorrente da mastite se deve à reduçáo da produçâao de leite, ao descarte de matrizes, à reduçâa do valor comercial desses animais, às perdas na evoluçáo genética do rebanho $\mathrm{e}$ aos gastos com medicamentos e mão de obra extra (DüRR et al., 2004). Além disso, a doença causa prejuízos à indústria de laticínios devido a alteraçóes na composição físico-química do leite, e ainda constitui ameaça à saúde dos consumidores devido à veiculaçấo de patógenos e suas toxinas, ou pela presença de resíduos de antibióticos no leite (Oliveira, 1999; Santos; Fonseca, 2007).

Embora haja grande diversidade entre os patógenos causadores de mastite em bovinos, S. aureus, Streptococcus agalactiae, Streptococcus dysgalactiae, Streptococcus uberis e Escherichia coli são responsáveis por cerca de 80\% dos casos (Ranjan et al., 2006). Individualmente, $S$. aureus destaca-se como um dos micro-organismos mais frequentemente encontrados nas infecçóes intramamárias (IIM) de bovinos em todos os continentes, e também é aquele que isoladamente determina as maiores perdas na pecuária leiteira (ZschöcK et al., 2000; SCHLEGelovÁ et al., 2003; VASUDEVAN et al., 2003). No Brasil, S. aureusé considerado como o principal agente causal da mastite bovina, com taxas de isolamento entre rebanhos que variam entre 8,3\% e 49,23\% (LANGONi et al., 1991; Costa et al., 1995; MoretTi et al., 1998; Brito et al. 1999; LAFFranchi et al., 2001; Donatele et al., 2002).

Entre as estratégias para controle das infecçóes estafilocócicas, destacam-se o tratamento precoce de casos clínicos e a terapia de vacas secas (Santos; FonSECA, 2007). Em ambos os casos, é necessário conhecer o perfil de resistência dos isolados visando à escolha dos antimicrobianos mais adequados para as referidas terapias, uma vez que os perfis de resistência de $S$. aureus podem ser bastante variáveis entre rebanhos, e até mesmo dentro de um mesmo rebanho (CARDoso et al., 2000; SiLVEIRA-FiLho et al., 2005; RABELLO et al., 2005). Diversos estudos apontam que este agente, além de ser o mais frequentemente isolado da mastite bovina no Brasil, apresenta aumento crescente no padráo de resistência a antimicrobianos (Brito et al., 2001). Segundo BARRAVIERA (1994), tal fato pode estar relacionado à capacidade diferenciada de aquisição de resistência que este micro-organismo possui.

O monitoramento da resistência em S. aureus é importante, pois o uso incorreto e indiscriminado de antimicrobianos é um dos principais fatores que influenciam no incremento das taxas de resistência. Tal conduta minimiza falhas terapêuticas e os riscos de desenvolvimento de resistência aos antimicrobianos, problemas bastante atuais nos campos da saúde humana e animal.
Desta forma, o presente estudo teve como objetivos determinar os perfis de resistência a antimicrobianos e avaliar a ocorrência do fenômeno de multirresistência em $S$. aureus isolados de infecçôes intramamárias de bovinos leiteiros no estado de Minas Gerais, Brasil, no período de 2004 a 2008.

\section{MATERIAL E MÉTODOS}

Foram utilizados no estudo 352 isolados caracterizados fenotípica e genotipicamente como $S$. aureus. Estes foram obtidos de amostras de leite assepticamente coletadas de animais acometidos pela mastite, provenientes de 35 rebanhos de bovinos leiteiros, totalizando 2.492 animais da regiáo sul do estado de Minas Gerais, no Brasil, no período de 2004 a 2008.

A caracterização fenotípica foi realizada por meio de testes de triagem que incluíram a morfologia macroscópica e microscópica, testes de catalase, oxidase, coagulase e produção de acetoína (teste de Voges Proskauer), realizados e interpretados segundo o National Mastitis Council (NMC, 2004). Isolados fenotipicamente identificados como $S$. aureus foram submetidos à identificação genotípica por meio da Reação em Cadeia da Polimerase (PCR) espécie-específica para o gene femA de $S$. aureus, de acordo com SiLva; SiLva (2005).

Amostras fenotípica e genotipicamente identificadas como S. aureus foram submetidas aos testes de suscetibilidade a antimicrobianos utilizando-se o método de difusão em disco, de acordo com as normas do Clinical Laboratory Standarts Institute (CLSI, 2008). Foram testados os antimicrobianos ampicilina (AMP), cefalotina (CFL), cefotaxima (CFX), cefoperazona (CPZ), ceftiofur (CFT), cloranfenicol (CLO), enrofloxacino (ENR), florfenicol (FLO), gentamicina (GEN), lincomicina (LIN), neomicina (NEO), nitrofurantoína NIT), novobiocina (NVB), polimixina $B(P O L)$, penicilina $G(P E N)$, oxacilina (OXA), sulfazotrim (SFZ), tetraciclina (TET) e cefquimona (CFQ), associaçōes de penicilina, nafcilina e dihidroestreptomicina (PND) e de neomicina, bacitracina e tetraciclina (NBT). A leitura e a interpretação dos resultados foram realizadas de acordo com os padróes do CLSI (2008) e do National Committee for Clinical Laboratory Standards (NCClsI, 2003). Para a interpretaçáo dos resultados para NBT e PND foram utilizados os parâmetros fornecidos pela MSD Saúde Animal, devido à inexistência de parâmetros para essas associaçóes no CSLI ou NCLSI. Amostras que se apresentaram moderadamente sensíveis aos antimicrobianos testados foram interpretadas como resistentes para a obtenção dos índices de resistência totais.

Os índices de resistência para cada antimicrobiano e o índice de múltipla resistência aos antimicrobianos (MAR) foram obtidos para todos os isolados testados de acordo com Krumperman (1983). Staphylococcus aureus ATCC 25923, Escherichia coli ATCC 25922 e Pseudomonas aeruginosa ATCC 27853 foram utilizados para controle dos testes de antibiograma. 


\section{RESULTADOS E DISCUSSÃO}

Os resultados dos testes de suscetibilidade foram compilados nas Figuras 1 e 2. Não foi observada resistência para nitrofurantoína ou para as associaçóes de neomicina, bacitracina e tetraciclina (NBT), assim como de penicilina, nafcilina e dihidroestreptomicina (PND). Baixos índices de resistência foram observados para enrofloxacino e florfenicol, com valores de 0,26 e $0,40 \%$, respectivamente, e no grupo das cefalosporinas, com valores de $0,0,28$ e $0,40 \%$ para cefquimona, cefalotina e ceftiofur, respectivamente. Entre os aminoglicosídeos, verificaram-se índices de resistência de 1,69\% para gentamicina e 3,35\% para neomicina. Isolados resistentes à penicilina $\mathrm{G}$, polimixina $\mathrm{B}$ e ampicilina e sensíveis às demais drogas testadas constituíram o padrão de resistência predominante, perfazendo 34,09\% dos isolados, com ampla disseminação $(73,68 \%)$ entre os rebanhos analisados. Verificou-se ainda que 16 amostras, representando 4,54\% dos isolados testados, distribuídas entre oito rebanhos, foram sensíveis a todos os antimicrobianos testados.

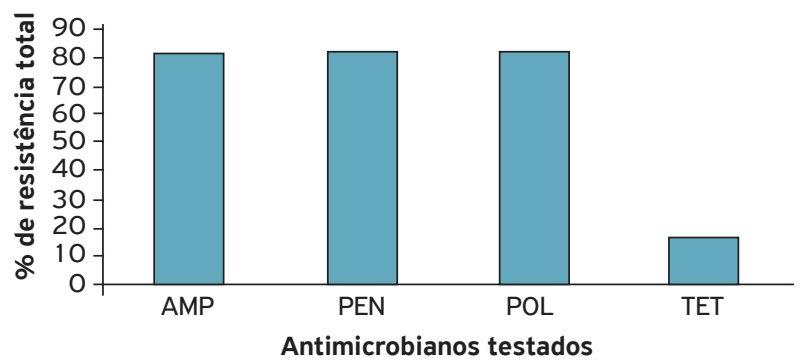

AMP: ampicilina; PEN: penicilina; POL: polimixina B; TET; tetraciclina.

Figura 1. Perfis de resistência a antimicrobianos testados para 352 amostras de Staphylococcus aureus isoladas de mastite em bovinos leiteiros do estado de Minas Gerais, no período de janeiro de 2004 a dezembro de 2008.
Embora as diferentes amostras apresentassem perfil de sensibilidade bastante similar para a maioria dos antimicrobianos testados, observaram-se, dentro do rebanho e entre rebanhos, variaçóes nos perfis de resistência para alguns produtos que são rotineiramente utilizados no tratamento da mastite bovina, tais como tetraciclina, lincomicina, cefoperazona e sulfazotrim. Em alguns rebanhos, foram observados até cinco padróes distintos de resistência, o que resultou em 54 padrôes de resistência diferentes para os 352 isolados testados.

Os maiores índices de resistência foram observados para polimixina B, penicilina e ampicilina, seguidas por lincomicina e tetraciclina (Fig. 1). Polimixina $B$ não é um antimicrobiano utilizado na rotina de tratamento da mastite, e seu uso é recomendado em casos de mastites ambientais ocasionadas por bactérias gram-negativas, devido à sua capacidade de inativar endotoxinas (LPS) (Mendes; Burdmann, 2009). A avaliação da suscetibilidade à polimixina $\mathrm{B}$ é uma prova utilizada para a diferenciação entre $S$. aureus (resistente) e Staphylococcus intermedius (sensível) (Quinn et al., 1994). No entanto, verificou-se neste estudo que aproximadamente $18 \%$ dos isolados de $S$. aureus foram suscetíveis a este antimicrobiano. Tal fato demonstra que este parâmetro fenotípico utilizado para a diferenciação de Staphylococcus spp. coagulase positiva (SCP) pode náo ser adequado para a caracterização de amostras de SCP isolados de mastite bovina.

Grandes diferenças quanto aos índices de resistência à penicilina têm sido observadas entre países. VINTOv et al. (2003) verificaram altas frequências de resistência à penicilina na Irlanda (71,4\%), Inglaterra $(67,3 \%)$ e nos Estados Unidos (50\%), enquanto frequências menores foram observadas nos países escandinavos, incluindo a Dinamarca (18,7\%), Noruega (2\%) e Suécia (28,5\%). РіткÄLÄ et al. (2004) verificaram que mais da metade das amostras de $S$. aureus isoladas de casos de mastite em rebanhos bovinos da Finlândia foram resistentes à penicilina G (52,1\%), índice significativamente maior que o registrado em outros países

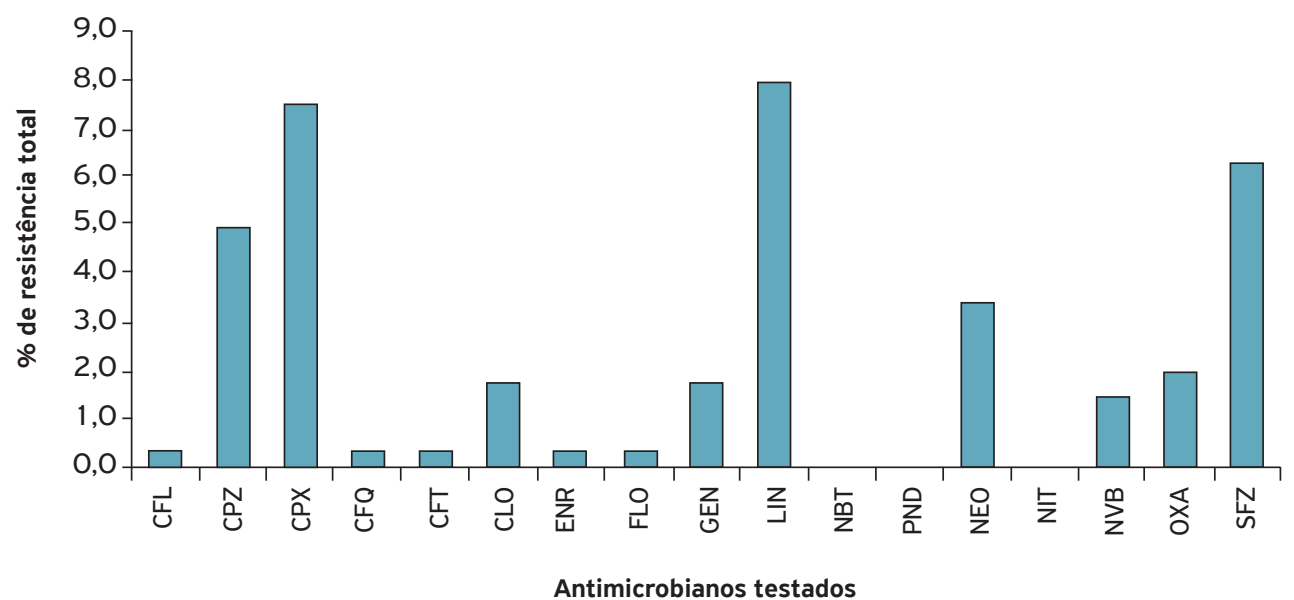

CFL: cefalotina; CPZ: cefoperazona; CFX: cefotaxima; CFQ: cefquimona; CFT: ceftiofur; CLO: cloranfenicol; ENR: enrofloxacino; FLO: florfenicol; GEN: gentamicina; LIN: lincomicina; NEO: neomicina; NIT: nitrofurantoína; NVB: novobiocina; OXA: oxacilina; SFZ: sulfazotrim; PND: associação de penicilina, nafcilina e dihidroestreptomicina; NBT: associação de neomicina, bacitracina e tetraciclina.

Figura 2. Perfis de resistência aos aos antimicrobianos testados para 352 amostras de Staphylococcus aureus isoladas de mastite em bovinos leiteiros do estado de Minas Gerais, no período de janeiro de 2004 a dezembro de 2008. 
nórdicos. Segundo esses autores, o índice elevado de resistência à penicilina e derivados na Finlândia está associado ao uso rotineiro de formulaçốes intramamárias para o tratamento da mastite naquele país, em contraste com as políticas rígidas de controle do uso de antimicrobianos em países nórdicos. Tal argumento também pode ser utilizado para justificar os elevados índices de resistência para penicilina G, ampicilina e tetraciclina observados em nosso estudo, tendo em vista o uso intensivo de preparaçóes contendo esses princípios ativos na rotina veterinária para o tratamento de infecções intramamárias em vacas secas ou lactantes, ou mesmo de outras patologias de causas infecciosas em rebanhos brasileiros.

Quanto ao índice de resistência antibiótica múltipla, o índice MAR, foram observadas variaçôes de 0 a 0,26 , com a média de 0,13 . Entre as amostras testadas, 65 , correspondendo a $18,15 \%$ dos estafilococos testados, oriundos de 24 entre os 35 rebanhos estudados, apresentaram índice MAR $\geq 0,2$, o que caracteriza o fenômeno da múltipla resistência segundo KRUMPERMAN (1983).

No que se refere à resistência aos antimicrobianos em amostras de $S$. aureus envolvidas na etiologia da mastite bovina em rebanhos brasileiros, diferentes pesquisadores (NADER FilHo et al., 1984; Riedner et al., 1987; Langoni et al., 1991; Donatele et al., 2002; RABello et al., 2005) relataram grande variação quanto à eficiência das diferentes drogas analisadas, conforme observado no presente trabalho. No entanto, algumas tendências podem ser percebidas nesses estudos, como a suscetibilidade da maioria dos isolados de $S$. aureus aos aminoglicosídeos, cefalosporinas e ao cloranfenicol, assim como a resistência à penicilina e à ampicilina.

Os resultados obtidos no presente estudo foram discrepantes daqueles obtidos por LANGE et al. (1999), que embora também tenham demonstrado altos índices de resistência à penicilina e à ampicilina para isolados de $S$. aureus provenientes de rebanhos leiteiros do sul do Brasil, diferentemente de nossos resultados, constataram que $48 \%$ foram sensíveis a todos os antibióticos testados. Também foram diferentes daqueles relatados por Silveira-Filho et al. (2005), que verificaram índices de resistência superiores a 50\% para gentamicina, lincomicina, tetraciclina e oxacilina em rebanhos leiteiros do estado de Pernambuco. Resistência à oxacilina e à gentamicina em $S$. aureus associada à mastite bovina foi incomum entre isolados testados no presente estudo e em relação a estudos prévios realizados em outras bacias leiteiras do Brasil (NADer Filho et al., 1984; Riedner et al., 1987; Langoni et al., 1991; Cardoso et al., 2000; Donatele et al., 2002; RABELLO et al., 2005).

Um estudo preliminar de avaliaçáo da suscetibilidade a antimicrobianos realizado com amostras de $S$. aureus isoladas de casos de mastite em rebanhos bovinos leiteiros de diferentes municípios do estado de Minas Gerais, no período de 1994 a 1997, realizado por CARDOso et al. (2000) apontou resultados muito semelhantes aos obtidos neste trabalho, tendo sido verificada alta suscetibilidade in vitro dos isolados à cefotaxima (100\%), enrofloxacina $(98,4 \%)$, gentamicina $(98,4 \%)$, cloranfenicol $(90,4 \%)$, sulfazotrim $(86,6 \%)$ e novobiocina $(85,8 \%)$, assim como elevada taxa de resistência para polimixina B (91,3\%), ampicilina $(71,4 \%)$ e penicilina $\mathrm{G}(70,9 \%)$. A análise comparativa dos resultados obtidos no presente trabalho em relaçáo aos obtidos por Cardoso et al. (2000) revela que não houve modificação apreciável no perfil de resistência da população de $S$. aureus envolvida na etiologia da mastite bovina após dez anos da realizaçáo do estudo no estado de Minas Gerais.

Estudos de perfis de resistência de $S$. aureus isolados de infecçōes intramamárias em bovinos, que foram realizados em outros países apontam, à semelhança dos resultados obtidos no presente trabalho, grande variação nos perfis de suscetibilidade aos antimicrobianos e a tendência de níveis elevados de resistência aos betalactâmicos. $\mathrm{Na}$ maioria desses estudos, a ocorrência frequente de resistência à penicilina tem sido relatada, enquanto a resistência a outros agentes antimicrobianos é menos comum (StePHAn et al., 2001; ERsKine et al., 2003; VinTov et al., 2003; NADER FilHo et al., 2007; SAEKI, 2011).

Na Argentina, Gentilini et al. (2000) avaliaram os perfis de suscetibilidade em 206 cepas de $S$. aureus isolados de mastite clínica e subclínica bovina entre 1996 e 1998. Os resultados foram muito semelhantes aos obtidos no presente estudo, verificandose que $83(40,3 \%)$ e $7(3,4 \%)$ dos isolados de $S$. aureus testados foram resistentes à penicilina e à gentamicina, respectivamente, e que nenhuma resistência foi observada para oxacilina e cefalotina. Os resultados obtidos no presente trabalho também foram semelhantes aos obtidos por Oliveira et al. (2000), ao estudarem os perfis de susceptibilidade de 811 cepas de $S$. aureus isoladas de casos de mastite bovina provenientes da Dinamarca (105), Inglaterra (92), Finlândia (95), Alemanha (103), Islândia (22), Irlanda (42), Noruega (101), Suécia (123), Suíça (69), Estados Unidos (53) e Zimbábue (6) a diferentes agentes antimicrobianos. Estes autores verificaram índices de resistência mais elevados para penicilina e derivados, e baixos índices de resistência para os demais agentes antimicrobianos testados, independentemente do país.

A terapia de vacas secas e o tratamento de casos clínicos constituem importantes ferramentas para o controle e a prevenção da mastite bovina (SANTOS; FonseCA, 2007). No entanto, o sucesso dessas medidas depende de vários aspectos, dentre eles, da escolha e da posologia adequada das drogas, da distribuiçáo das drogas dentro da glândula mamária, do estado fisiológico do animal, da precocidade com que o tratamento é instituído e do micro-organismo envolvido (Gruet et al., 2001; Erskine et al., 2003). Desta forma, é imprescindível identificar os patógenos presentes e traçar o perfil de sensibilidade dos mesmos aos antimicrobianos, uma vez que micro-organismos de uma mesma espécie, mesmo dentro de um mesmo rebanho, podem ter perfis de sensibilidade diferentes (MCKelLar, 1991).

A resistência aos antimicrobianos é um fator importante no estabelecimento e na disseminação de clones bacterianos em um rebanho. Trata-se de um fenômeno bastante dinâmico, o qual tem estreita associação com mudanças no manejo, tais como o uso sistemático de antimicrobianos e o uso da ordenhadeira mecânica, 
considerados importantes fatores que atuam como forças seletivas sobre patógenos causadores de mastite (MYLlys et al., 1994).

Os resultados obtidos demonstraram variaçóes nos perfis de resistência dos isolados, entre rebanhos e dentro de rebanhos, bem como a ocorrência de amostras com múltipla resistência nos rebanhos testados. Estes resultados apontam a necessidade de se monitorar periodicamente o perfil de suscetibilidade dos diferentes micro-organismos envolvidos na etiologia da mastite bovina, visando acompanhar a evolução dos índices de resistência e para a escolha das drogas mais adequadas a serem utilizadas, preventiva e terapeuticamente.

\section{CONCLUSÕES}

Os testes de suscetibilidade aos antimicrobianos apontaram grande variação nos perfis de resistência dos isolados entre rebanhos e dentro de rebanhos.

Isolados resistentes à penicilina $\mathrm{G}$, à polimixina $\mathrm{B}$ e à ampicilina sensíveis às demais drogas testadas constituíram o padrão de resistência predominante. O fenômeno da múltipla resistência aos antimicrobianos foi observado em baixa proporção, demonstrando que o problema ainda não é tão relevante entre $S$. aureus isolados de mastite bovina.

\section{REFERÊNCIAS}

AARESTRUP, F.M.; JENSEN, N.E. Prevalence and duration of intrammamary infection in danish heifers during the peripartum period. Journal of Dairy Sciences, v.80, n.2, p.307-312, 1997.

BARRAVIERA, B. Estudo clínico das estafilococcias. Jornal Brasileiro de Medicina, Rio de Janeiro, v.67, n.2, p.160-192, 1994.

BRADLEY, A.J. Bovine mastitis: an evolving disease. Veterinary Journal, v.164, n.2, p.116-128, 2002.

BRITO, M.A.V.P.; BRITO, J.R.F.; RIBEIRO, M.T.; VEIGA, V.M.O. Padrão de infecção intramamária em rebanhos leiteiros: exame de todos os quartos mamários de vacas em lactação. Arquivo Brasileiro de Medicina Veterinária e Zootecnia, v.51, p.129135, 1999.

BRITO, M.A.V.P.; BRITO, J.R.F.; SILVA, M.A.S.; CARMO, R.A. Concentração mínima inibitória de dez antimicrobianos para amostras de $S$. aureus isoladas de infecção intramamária bovina. Arquivos Brasileiros de Medicina Veterinária e Zootecnia, v.53, n.5, p.10-17, 2001.

CARDOSO, H.F.T.; COSTA, G.M.; SILVA, N. Susceptibilidade a antimicrobianos de Staphylococcus aureus isolados de leite bovino no Estado de Minas Gerais. Revista Brasileira de Medicina Veterinária, v.22, n.5, p.199-206, 2000.

CLSI (3aed). Performance standards for antimicrobial disk susceptibility tests for bacteria isolated from animals; Approved standard. v.28, n.8 - CLSI document M31-A3, 2008. 99p.

COSTA, E.O.; MELVILLE, P.A.; RIBEIRO, A.R.; WATANABE, E.T.; WHITE, C.R.; PARDO, R.B. Índices de mastite bovina clínica e subclínica nos estados de São Paulo e Minas Gerais. Revista Brasileira de Medicina Veterinária, v.17, n.5, p.215-217, 1995.

DONATELE, D.M.; MOTTA, O.V.; FOLLY, M.M. Perfil antimicrobiano de linhagens de Staphylococcus spp.coagulase positiva na mastite subclínica de vacas leiteiras nas regiões norte e noroeste do Estado do Rio de Janeiro. Revista NAPGAMA, v.5, n.2, p.3-6, 2002.
DÜRR, J.W.; CARVALHO, M.P.; SANTOS, M.V.O compromisso com a qualidade do leite no Brasil. Passo Fundo: Editora UPF, 2004. p.38-55.

ERSKINE, R.J.; WAGNER, S.; DEGRAVES, F.J. Mastitis therapy and pharmacology. Veterinary Clinical Food Animal Practice, v.19, p. 109-138, 2003.

FAGUNDES, H.; OLIVEIRA, C.A.F. Infecções intramamárias causadas por Staphylococcus aureus e suas implicações em saúde pública. Ciência Rural, v.34, n.4, p.1315-1320, 2004.

GENTILINI, E.; DENAMIEL, G.; LLORENTE, P.; GODALY, S.; REBUELTO, M.; DEGREGORIO, O. Antimicrobial susceptibility of Staphylococcus aureus isolated from bovine mastitis in Argentina. Journal of Dairy Sciences, v.83, p.1224-1227, 2000.

GIGUĖRE, S.; PRESCOTT, J.F.; BAGGOT. J.D.; WALKER, R.D.; DOWLING, P.M. Terapia antimicrobina em medicina veterinária (4a ed.). São Paulo: Editora Roca, 2010. 683p.

GRUET, P.; MAINCENT, P.; BERTHELOT, X.; KALTSATOS, V. Bovine mastitis and intramammary drug delivery: review and perspectives. Advanced Drug Delivery Reviews, v.50, p.245-259, 2001.

KRUMPERMAN, P.H. Multiple antibiotic resistance indexing of Escherichia coli to identify high-risk sources of fecal contamination of foods. Applied and Environmental Microbiology, v.46, n.1, p. $1165-70,1983$.

LAFFRANCHI, A.; MULLER, E. E.; FREITAS, J. C. Etiologia das infecções intramamárias em vacas primíparas ao longo dos primeiros quatro meses de lactação. Ciência Rural, v.31, n.6, p.1027-1032, 2001.

LANGE, C.; CARDOSO, M.; SENCZEK, D.; SCHWARZ, S. Molecular subtyping of Staphylococcus aureus isolated from cases of bovine mastitis in Brazil. Veterinary Microbiology, v .67, p.127-141, 1999.

LANGONI, H.; PINTO, M.P.; DOMINGUES, P.F.; LISTONI, F.J.P. Etiologia e sensibilidade bacteriana da mastite bovina subclínica. 
Arquivo Brasileiro de Medicina Veterinária e Zootecnia, v.43, n.6, p.507-515, 1991.

LEBLANK, S.J.; LISSEMORE, K.D.; KELTON, D.F.; DUFFIELD, T.F.; LESLIE, K.E. Major advances in diseases prevention in dairy cattle. Jornal of Dairy Sciences, v.89, p. 1267-1279, 2006.

MENDES, C.AC.; BURDMANN, E. Polimixinas - revisão com ênfase na sua nefrotoxicidade. Revista da Associação Médica Brasileira, v.55, n.6, p.752-759, 2009.

McKELLAR, Q.A. Intramammary treatment of mastitis in cows. In Practice, p.244-249, 1991.

MORETTI, A.; PASQUALI, P.; MENCARONI, G.; BONCIO, B.; FIORETTI, D.P. Relationship between cell counts in bovine milk and the presence of mastitis pathogens (yeasts and bacteria). Journal of Veterinary Medicine, Series B, v.45, p.129-132, 1998.

MYLLYS, V.; HOKANEN-BUZALSKI, T.; HUOVINEN, P.; SANDHOLM, M. NURMI, E. Association of changes in the bacterial ecology of bovine mastitis with changes in the use of milking machines and antibacterial drugs. Acta Veterinaria Scandinavica, v.35, n.4, p.363369, 1994.

NADER FILHO, A.N.; ITURRINO, R.P.S.; ROSSI-JUNIOR, O.D. Mastite subclínica em rebanhos produtores de leite gordura 3,2\%. Arquivo Brasileiro de Medicina Veterinária e Zootecnia, v.36, n.5, p.549-58, 1984.

NADER FILHO, A.N.; FERREIRA, L.M.; DO AMARAL, L.A. ROSSI JUNIOR, O.D.; OLIVEIRA, R.P. Sensibilidade antimicrobiana dos Staphylococcus aureus isolados no leite de vacas com mastite. Arquivos do Instituto Biológico, v.74 n. 1 , p. $1-4,2007$

NCCLS. Performance standards for antimicrobial disk susceptibility tests; approved standard ( $8^{a}$ Ed.). Wayne, Pennsylvania: NCCLS, 2003.

NMC-National Mastitis Council. Microbiological procedures for diagnosis of bovine udder infections and determination of milk quality (4a Ed.). Verona: NMC, 2004. 47 p.

OLIVEIRA, A. P.; WATTS, J. L.; SALMON, S. A.; AARESTRUP,F. M. Antimicrobial susceptibility of Staphylococcus aureus isolated from bovine mastitis in Europe and the United States. Journal of Dairy Sciences, v.83, p.855-862, 2000.

OLIVEIRA, M.C.S. Manejo sanitário em sistemas intensivos de produção de leite. (Embrapa Pecuária Sudeste. Circular técnica, 18). São Carlos: EMBRAPA - CPPSE, 1999. 22p.

PITKÄLÄ, A., HAVERI, M; PYO, S.; LA, RA; MYLLYS, V.; HONKANENBUZALSKI, T. Bovine mastitis in Finland 2001 - Prevalence, distribution of bacteria, and antimicrobial resistance. Journal of Dairy Sciences, v.87, p.2433-2441, 2004.

QUINN, P.J.; CARTER, M.E.; MARKEY, B. Clinical veterinary microbiology. London: Wolfe, 1994, 648p.
RABELLO, R.F.; SOUZA, C.R.V.M.; DUARTE, R.S.R.; LOPES, M.M.; TEIXEIRA, L.M.; CASTRO, A.C.D. Characterization of Staphylococcus aureus isolates recovered from bovine mastitis in Rio de Janeiro, Brazil. Journal of Dairy Sciences, v.88, p.3211-3219, 2005.

RANJAN, R.; SWARUP, D.; PATRA, R.C. Bovine protothecal mastitis: a review. Perspectives in Agriculture, Veterinary Sciences, Nutrition and Natural Resources, v. 1, n. 17, p.1-7, 2006.

RIEDNER, S.; ALBUQUERQUE, A.J.D.; BADKE. M.R.T. Prevalência da mastite em dois tambos de Santa Maria-RS. Revista Científica do Centro de Ciências Rurais, v.17, p.261273, 1987.

SAEKI, E.K.; MELLO PEIXOTO, E.C.T.; MATSUMOTO, L.S.; MARCUSSO, P.F.; , MONTEIRO, R.M. Mastite bovina por Staphylococcus aureus: sensibilidade às drogas antimicrobianas e ao extrato alcoólico de própolis. Acta Veterinaria Brasilica, v.5, n.3, p.284-290, 2011.

SANTOS, M.V; FONSECA, L.F.L. Estratégias para controle da mastite e melhoria da qualidade do leite. São Paulo: Editora Manole Ltda, 2007, 314p.

SCHLEGELOVÁ, J.; DENDIS, M.; BENEDÍK, J.; BABÁK, V.; RYSÁNEK, D. Staphylococcus aureus isolates from dairy cows and humans on far differ in coagulase genotype. Veterinary Microbiology, v.92, p.327-334, 2003.

SILVA, E. R.; SILVA, N.Coagulase gene typing of Staphylococcus aureus isolated from cows with mastitis in southeastern Brazil. Canadian Journal of Veterinary Research, v.69, n.4, p.260-64, 2005.

SILVEIRA-FILHO, V.M.; SANTOS, F.G.B.; FREITAS, M.L.F.; LUZ, I.S.; ALMEIDA, A.M.P.; LELA, N.C; SENA, M.J.; MOTA, R.A.; LEALBALBINO, T.C. Molecular epidemiologic study of Staphylococcus aureus associated to bovine mastitis from Pernambuco state, Brazil. Revista NAPGAMA, v.8, n. 1, p.12-17, 2005.

STEPHAN, R.; ANNEMÜLLER, C.; HASSAN, A.A.; LÄMMLER, C. Characterization of enterotoxigenic Staphylococcus aureus isolated from bovine mastitis in north-east Switzerland. Veterinary Microbiology, v.78, p.373-382, 2001.

VASUDEVAN, P.; NAIR, M.K.M.; ANNAMALAI, T.A. Phenotypic and genotypic characterization of bovine mastitis isolates of Staphylococcus aureus for biofilm formation.Veterinary Microbiology, v.92, p.179-185, 2003.

VINTOV, J.; AARESTRUP, F.M.; ZINN, C.E.; OLSEN, J.E. Association between phage types and antimicrobial resistance among bovine Staphylococcus aureus from 10 countries. Veterinary Microbiology, v.95, p.133-147, 2003.

ZSCHÖCK, M.; BOTZLER, D.; BLÖCHER, S.; SOMMERHÄUSEN, J.; HAMANN, H. P. Detection of genes for enterotoxins (ent) and toxic shock syndrome toxin-1 (tst) in mammary isolates of Staphylococcus aureus by polymerase-chain-reaction. International Dairy Journal, v. 10, p. 569-574, 2000. 